\title{
In-Situ Atomic Scale Visualization of Battery Charging and Discharging
}

Jian Yu Huang ${ }^{1}$, Li Zhong ${ }^{2}$, Chong Min Wang ${ }^{3}$, John P. Sullivan ${ }^{1}, \mathrm{Wu} \mathrm{Xu}{ }^{3}$, Li Qiang Zhang ${ }^{2}$, Scott X. $\mathrm{Mao}^{2}$, Nicholas S. Hudak ${ }^{1}$, Xiao Hua Liu ${ }^{1}$, Arunkumar Subramanian ${ }^{1}$, Hong You Fan ${ }^{1}$, Liang Qi ${ }^{4}$, Akihiro Kushima ${ }^{4}, \mathrm{Ju} \mathrm{Li}^{4}$

${ }^{1}$ Sandia National Laboratories, Albuquerque, New Mexico 87185

${ }^{2}$ University of Pittsburgh, Pittsburgh, Pennsylvania 15261

${ }^{3}$ Pacific Northwest National Laboratory, Richland, Washington 99354

${ }^{4}$ Department of Materials Science and Engineering, University of Pennsylvania, Philadelphia, Pennsylvania 19104

Lithiation/delithiation of the electrode materials in Li-ion batteries (LIBs) induces large strains in the host material leading to plasticity and fracture. Lithiation is also often accompanied by phase transformations, such as electrochemically-driven solid-state amorphization (ESA). These electrochemical reaction-induced microstructural events limit the energy capacity and cycle lifetime of LIBs. It was recently reported that lithium-ion anode materials composed of nanowires can offer improved performance and lifetime compared to those of micron-scale or larger materials. The improvements are often attributed to the nanowire's unique geometry and enhanced accommodation of the transformation strains that occur during cycling. However, the detailed mechanisms of strain-induced plasticity and strain accommodation in nanowires during electrochemical charging are largely unknown.

We report the creation of a nanoscale electrochemical device inside a transmission electron microscope consisting of a single $\mathrm{SnO}_{2}$ nanowire anode, an ionic liquid electrolyte and a bulk $\mathrm{LiCoO}_{2}$ cathode - and the in-situ observation of the lithiation of the $\mathrm{SnO}_{2}$ nanowire during electrochemical charging [1]. Upon charging, a reaction front propagated progressively along the nanowire, causing the nanowire to swell, elongate, and spiral (Fig. 1). The reaction front is a "Medusa zone" containing a high density of mobile dislocations, which are continuously nucleated and absorbed at the moving front (Fig. 2). This dislocation cloud indicates large in-plane misfit stresses and is a structural precursor to electrochemically-driven solid-state amorphization. Because lithiation induced volume expansion, plasticity and pulverization of electrode materials are the major mechanical effects that plague the performance and lifetime of high capacity anodes in lithium-ion batteries, our observations provide important mechanistic insight for the design of advanced batteries.

\section{References}

1. J.Y. Huang et al., Science 330, 1515-1520 (2010); Y. M. Chiang, Science 330, 1485 (2010

This work was performed, in part, at the Center for Integrated Nanotechnologies, a U.S. Department of Energy, Office of Basic Energy Sciences user facility. Sandia National Laboratories is a multi-program laboratory operated by Sandia Corporation, a Lockheed-Martin Company, for the U. S. Department of Energy under Contract No. DE-AC04-94AL85000. 


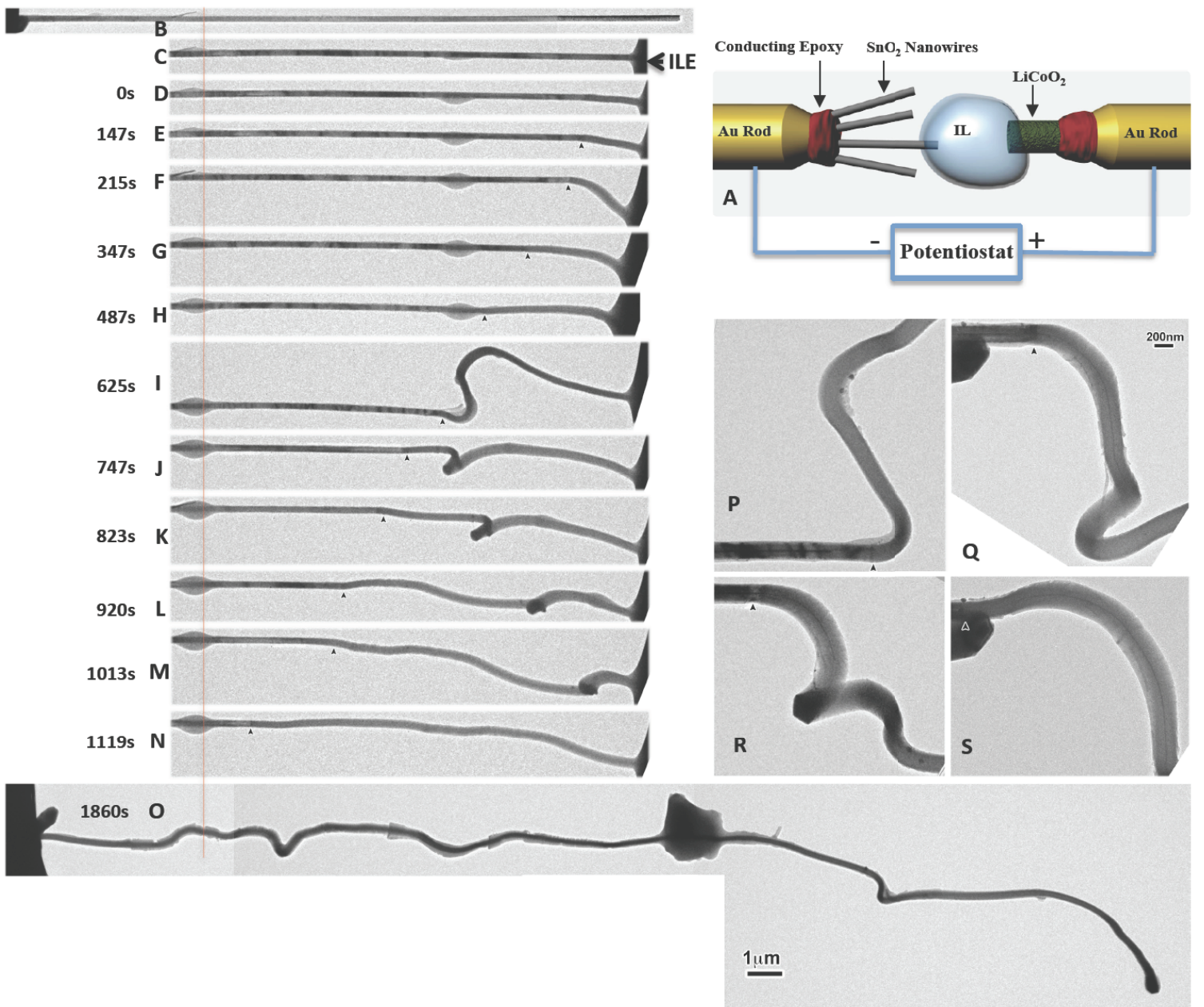

Fig. 1 Time lapse structure evolution of a $\mathrm{SnO}_{2}$ nanowire anode during charging at $-3.5 \mathrm{~V}$ against a $\mathrm{LiCoO}_{2}$ cathode. The single crystal nanowire was elongated $60 \%$ and the diameter increased $45 \%$ (resulting in a $240 \%$ volume expansion) following charging for $1860 \mathrm{~s}$. (A) Schematic of the experimental setup. The initially straight nanowire (B and $\mathbf{C}$ ) became significantly twisted and bent after charging (D to $\mathbf{S}$ ). The chemical reaction front progressed along the nanowire's longitudinal direction, with the front clearly visible, as pointed out by arrowheads in $(\mathbf{E})$ to $(\mathbf{S})$. The red line in $(\mathrm{B})$ to $(\mathrm{O})$ marks a reference point to track the change of the nanowire length. (P) to $(\mathbf{S})$ are sequential high magnification images showing the progressive migration of the reaction front, swelling, and the twisted morphology of the nanowire after the reaction front passed by.

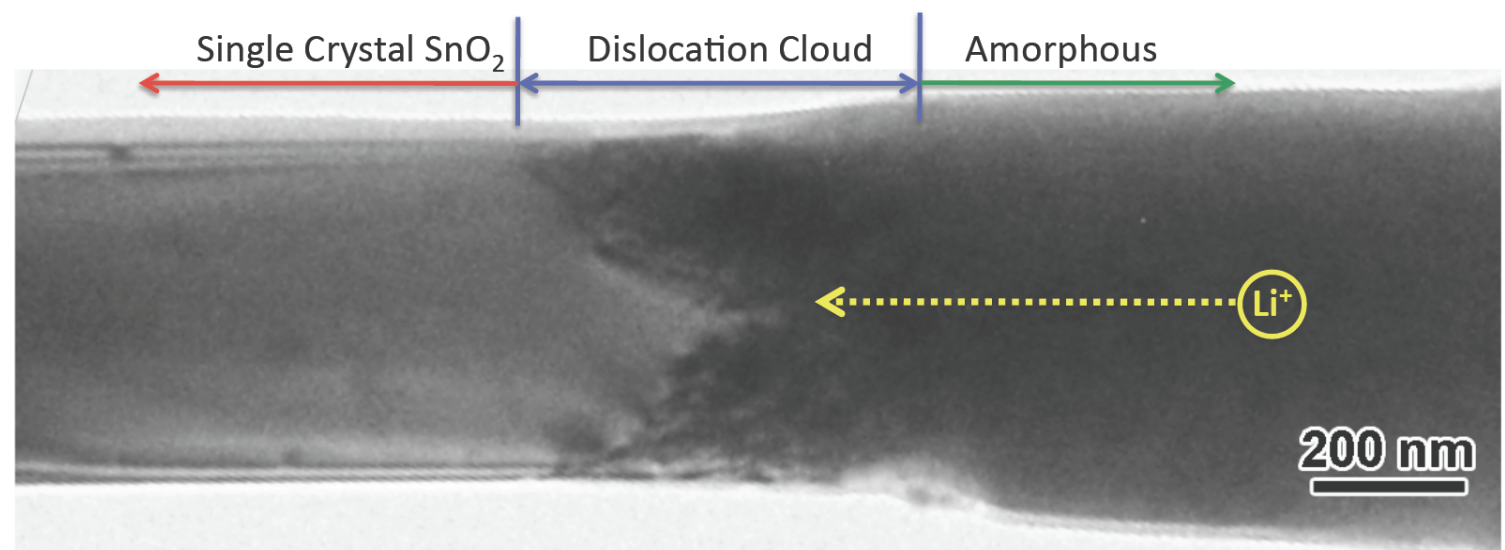

Fig. 2 The reaction front contains high density of dislocations. 